\title{
Chirality-Induced Phonon Dispersion in a Noncentrosymmetric Micropolar Crystal
}

\author{
J. Kishine $\odot,{ }^{1,2}$ A. S. Ovchinnikov $\oplus^{3,4}$ and A. A. Tereshchenko ${ }^{3}$ \\ ${ }^{1}$ Division of Natural and Environmental Sciences, The Open University of Japan, Chiba, 261-8586, Japan \\ ${ }^{2}$ Institute for Molecular Science, Okazaki, Aichi 444-8585, Japan \\ ${ }^{3}$ Institute of Natural Sciences and Mathematics, Ural Federal University, Ekaterinburg 620083, Russia \\ ${ }^{4}$ Institute of Metal Physics, Ural Division, Russian Academy of Sciences, Ekaterinburg 620219, Russia
}

(Received 15 July 2020; accepted 4 November 2020; published 9 December 2020)

\begin{abstract}
Features of the phonon spectrum of a chiral crystal are examined within the micropolar elasticity theory. This formalism accounts for not only translational micromotions of a medium but also rotational ones. It is found that there appears the phonon band splitting depending on the left- and right-circular polarization in a purely phonon sector without invoking any outside subsystem. The phonon spectrum reveals parity breaking while preserving time-reversal symmetry, i.e., it possesses true chirality. We find that hybridization of the microrotational and translational modes gives rise to the acoustic phonon branch with a "roton" minimum reminiscent of the elementary excitations in the superfluid helium- 4 . We argue that a mechanism of this phenomena is in line with Nozières' reinterpretation P. Nozières, [J. Low Temp. Phys. 137, 45 (2004)] of the rotons as a manifestation of an incipient crystallization instability. We discuss a close analogy between the translational and rotational micromotions in the micropolar elastic medium and the Bogoliubov quasiparticles and gapful density fluctuations in ${ }^{4} \mathrm{He}$.
\end{abstract}

DOI: 10.1103/PhysRevLett.125.245302

Introduction.-In the hierarchy of electric, magnetic, and mechanical degrees of freedom and attendant interrelationships, mechanical and elastic properties of solids are most sensitive to structure. This is why the mechanical response in solids has recently been the target of a new branch of electronics, referred to as straintronics [1]. The connection of structural chirality with static and dynamical properties offers a key to understanding the functionality of chiral systems [2]. A quintessential example of the chiralitycontrolled phenomenon is the optical activity [3], where the propagation of circularly polarized light through a chiral material depends on its handedness. As for elastic degrees of freedom, a mechanical counterpart of optical activity, so-called acoustical activity, has been attracting revived attention [4]. This phenomenon was first predicted by Portigal and Burstein [5] and direct observation thereof was subsequently provided for the $\alpha$-quartz crystal belonging to an enantiomorphic space group [6].

The acoustical activity is also related with first-order spatial dispersion contributions to the elastic constants [5]. This situation is somewhat similar to the role of Dzyaloshinskii-Moriya interaction which leads to a linear Lifshitz invariant in free energy of a chiral helimagnet [7,8]. However, chirality effects are beyond the conventional elasticity theory [9], which considers only a local translation of points and the force stress (force per unit area) but completely ignores a local rotation of these points and the concomitant couple stress (a torque per unit area). As a consequence, there appears the elastic four-rank tensor $C_{i j k l}$, which connects the parity-even and parity-odd second rank tensors, and gives rise to the chiral term in the energy functional $\left(C_{k l m n} \varepsilon_{k l} \gamma_{m n}\right.$ as explained below).

These missing effects, which may be viewed as a particular manifestation of nonlocality, are addressed in the micropolar elasticity theory $[10,11]$. So far, only a few attempts have been made to calculate dispersion curves of the micropolar elastic waves in crystals. We note in this regard the pioneering research undertaken by Pouget $e t$ al. for the centrosymmetric compound $\mathrm{KNO}_{3}$ [12], where the spectrum of the micropolar waves reciprocal in the momentum space was obtained.

Then, the natural question arises as to whether or not a phonon spectrum in a chiral crystal exhibits nonreciprocity effects in the propagation of micropolar elastic waves. This is the question addressed in this Letter. A salient feature of the nonreciprocity is a polarizationdependent splitting of phonon bands similar to the one for electronic bands due to spin-orbit coupling [13]. In contrast to the phonon magnetochiral effect $[14,15]$, for which the splitting is achieved through coupling with nonreciprocal magnons, our aim is to find the result for a purely phononic sector without the involvement of any subsystem outside.

Another issue discussed in this Letter is how the structural chirality relates to the phonon angular momentum and spin. In this regard, we mention the recent studies of chiral phonons in monolayers of hexagonal [16] and kagome lattices [17]. In these systems, the phonon eigenmodes at high-symmetry points of the Brillouin zone inherit the threefold rotational symmetry of the lattice 


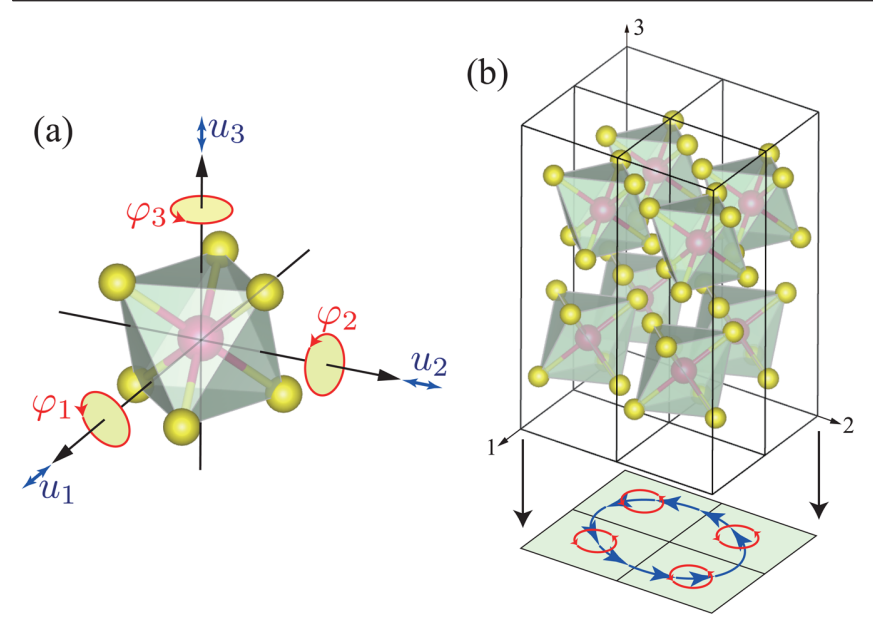

FIG. 1. (a) Schematic view of the rotational and translational degrees of freedom of the atomic microelement. As an example of the microelement, we show the $\mathrm{CrS}_{6}$ block inside the elementary cell of $\mathrm{CrNb}_{3} \mathrm{~S}_{6}$. (b) Typical distribution of microrotation fields. We also depict conceptual representation of the microrotation (red small circle with arrows) and translation (blue large circle with arrows) associated with a circularly polarized mode, which are projected on the 1-2 plane.

which allows labeling these phonon eigenmodes with pseudoangular momentum. It includes both orbital and spin parts, the latter coincides with the phonon chirality characterized by the circular polarization of phonons. In contrast to this scenario in which chirality is assigned only to special Brillouin zone points, we examine an effect of the structural chirality on phonon dispersion over the whole Brillouin zone. The chiral helimagnet $\mathrm{CrNb}_{3} \mathrm{~S}_{6}$ serves to illustrate our results, one of which is the striking similarity of microrotations embedded in the micropolar elasticity theory with roton excitations in helium-4.

Chiral phonon dispersions for the point group 622.-A general scheme of treatment of elementary excitations in the context of the micropolar elasticity theory may be found in Supplemental Material [18]. In this theory, the field of translational displacements $\boldsymbol{u}(\boldsymbol{r})$ is supplemented by the field of microrotations $\varphi(\boldsymbol{r})$, and both are attributed to a microelement located at the position $\boldsymbol{r}$ (see Fig. 1). Two linear micropolar strain tensors $\varepsilon_{k l}=\partial_{l} u_{k}-\epsilon_{k l m} \varphi_{m}$ and $\gamma_{k l}=\partial_{l} \varphi_{k}$ form measures of microdeformations. Here and throughout, $\epsilon_{k l m}$ is the Levi-Civita symbol and the Einstein summation convention is used. The strain energy density is given by the quadratic form $U=\frac{1}{2} A_{k l m n} \varepsilon_{k l} \varepsilon_{m n}+\frac{1}{2} B_{k l m n} \gamma_{k l} \gamma_{m n}+C_{k l m n} \varepsilon_{k l} \gamma_{m n}$, where the third term (chiral term) changes its sign under the inversion operation while the first and the second do not. This chiral coupling leads to the left- and right-circularpolarization-depdendent nonreciprocal phonon dispersion.

To demonstrate how the chiral coupling gives rise to new peculiarities of phonon dispersion, we consider, as an example, the layered compound $\mathrm{CrNb}_{3} \mathrm{~S}_{6}$ which has the noncentrosymmetric hexagonal space group $P 6_{3} 22$. In this material, the $\mathrm{Cr}$ atoms are intercalated between the sandwich layers $\mathrm{S}-\mathrm{Nb}-\mathrm{S}$ of the disulfide $\mathrm{NbS}_{2}$ and surrounded by the six $\mathrm{S}$ atoms in an octahedral geometry. The $\mathrm{CrS}_{6}$ octahedra are not linked to each other. The distance $d(\mathrm{Cr}-\mathrm{S})=2.393 \AA[21]$ is less than $\sim 0.1 \AA$ the distance $d(\mathrm{Nb}-\mathrm{S})=2.47-2.50 \AA[22]$. Assuming that modes due to relative displacements of the $\mathrm{Cr}$ and $\mathrm{S}$ ions are not excited, the $\mathrm{CrS}_{6}$ may be modeled as a rigid structural unit. Below, we consider plane waves propagating along the crystalline [001] axis, i.e., the chiral axis.

Dispersion of the micropolar waves is obtained in the following way. To describe transverse modes, it is appropriate to introduce the circular basis $u_{ \pm}=u_{1} \pm i u_{2}$ and $\varphi_{ \pm}=\varphi_{1} \pm i \varphi_{2}$, where + and - correspond to the left or right circularly polarized microdeformation fields. Then, the equations of motion (EOMs) for the decoupled transverse modes may be written as

$$
\begin{aligned}
\rho \ddot{u}_{ \pm}= & A_{55} \partial_{3}^{2} u_{ \pm}+C_{74} \partial_{3}^{2} \varphi_{ \pm} \mp i\left(A_{47}-A_{55}\right) \partial_{3} \varphi_{ \pm}, \quad \text { (1) } \\
\rho j_{ \pm} \ddot{\varphi}_{ \pm}= & C_{74} \partial_{3}^{2} u_{ \pm} \mp i\left(A_{47}-A_{55}\right) \partial_{3} u_{ \pm}+B_{44} \partial_{3}^{2} \varphi_{ \pm} \\
& \mp 2 i\left(C_{44}-C_{74}\right) \partial_{3} \varphi_{ \pm}-\left(A_{44}-2 A_{47}+A_{55}\right) \varphi_{ \pm},
\end{aligned}
$$

where $\rho=5.029 \mathrm{~g} / \mathrm{cm}^{3} \quad[23]$ and $j_{ \pm}=j_{11}=0.5 \times$ $10^{-19} \mathrm{~m}^{2}$ [24] are the mass density and the microinertia tensor component, respectively. Here, the four-rank tensor elements are represented in the Sirotin (generalized Voigt) scheme [18]. The most important point is the appearance of the linear gradient terms $\partial_{3} u_{ \pm}$and $\partial_{3} \varphi_{ \pm}$, which cause polarization-dependent velocities. Hybridization of these circularly polarized $u_{ \pm}$and $\varphi_{ \pm}$mode gives rise to acoustic and optical branches of the spectrum of propagating transverse waves in the micropolar medium. It is to be noted that each of the modes, $u_{ \pm}$( or $\varphi_{ \pm}$), is nonreciprocal in real space, but they form a pair invariant under timereversal symmetry. This may be inferred from Eqs. (1) and (2) which break parity $\mathcal{P}$ but preserve time-reversal symmetry $\mathcal{T}$. This situation means that phonons in the micropolar chiral crystal exhibit true chirality in contrast to the phonon [14,15] or electrical [25] magnetochiral effects, where both $\mathcal{P}$ and $\mathcal{T}$ are simultaneously broken. On that understanding, we call these excitations truly chiral phonons. This polarization-dependent splitting of the transverse phonon branches is analogous to the Rashba splitting of electronic bands, in which $\mathcal{P}$ is also broken but not $\mathcal{T}$. Nonreciprocity of the truly chiral phonons originates from the $\left(C_{44}-C_{74}\right)$ coupling term in the EOMs, Eqs. (1) and (2), and vanishes for $C_{44}=C_{74}$.

The equations that govern propagation of the longitudinal branches are written as

$$
\rho \ddot{u}_{3}=A_{33} \partial_{3}^{2} u_{3}+C_{33} \partial_{3}^{2} \varphi_{3},
$$




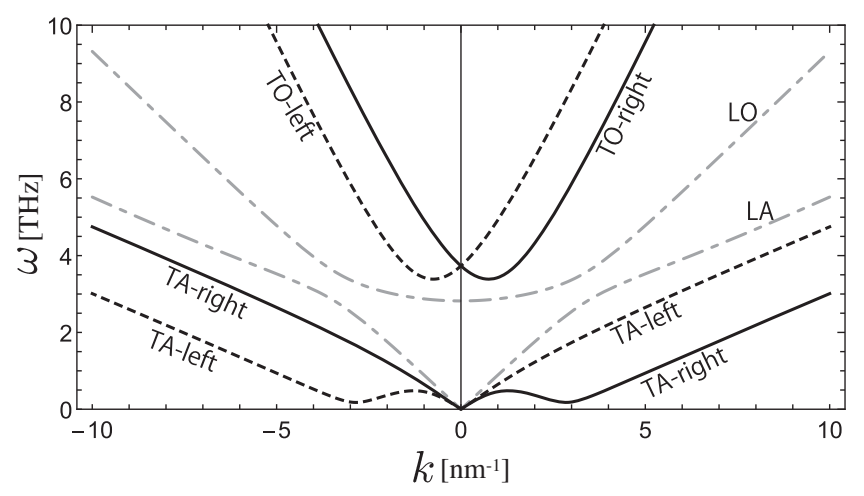

FIG. 2. Phonon dispersion curves for the chiral micropolar crystal: the longitudinal acoustic (LA) and optical (LO) branches (dash-dotted line), the transverse left-handed acoustic (TA-left) and optical (TO-left) branches (dashed line), the transverse right-handed acoustic (TA-right) and optical (TO-right) branches (solid line). Numerical values of the tensor components are chosen as $A_{33}=0.4 \times 10^{10} \mathrm{~N} / \mathrm{m}^{2}, A_{66}=4.9 \times 10^{10} \mathrm{~N} / \mathrm{m}^{2}$, $A_{69}=4.7 \times 10^{10} \mathrm{~N} / \mathrm{m}^{2}, B_{33}=1.5 \times 10^{-10} \mathrm{~N}, C_{33}=0.3 \mathrm{~N} / \mathrm{m}$ for the longitudinal modes, and $A_{44}=0.21 \times 10^{10} \mathrm{~N} / \mathrm{m}^{2}$, $A_{55}=0.215 \times 10^{10} \mathrm{~N} / \mathrm{m}^{2}, \quad A_{47}=0.195 \times 10^{10} \mathrm{~N} / \mathrm{m}^{2}, \quad B_{44}=$ $1.0 \times 10^{-10} \mathrm{~N}, C_{44}=0.44 \mathrm{~N} / \mathrm{m}, C_{74}=0.36 \mathrm{~N} / \mathrm{m}$ for the transverse modes, respectively.

$$
\rho j_{3} \ddot{\varphi}_{3}=C_{33} \partial_{3}^{2} u_{3}+B_{33} \partial_{3}^{2} \varphi_{3}-2\left(A_{66}-A_{69}\right) \varphi_{3},
$$

where $j_{3}=j_{33}$ will be taken as $1.0 \times 10^{-19} \mathrm{~m}^{2}$. Note the absence of linear gradient terms that entails simple hybridization of the $u_{3}$ and $\varphi_{3}$ modes.

Phonon dispersion relations may be obtained inserting plane waves propagating along the chiral $\left(x_{3}\right)$ axis, $u_{\alpha}\left(x_{3}, t\right)=u_{\alpha} e^{i\left(k x_{3}-\omega t\right)}$ and $\varphi_{\alpha}\left(x_{3}, t\right)=\varphi_{\alpha} e^{i\left(k x_{3}-\omega t\right)}$, into Eqs. (1)-(4). Hereinafter, the index $\alpha$ labels either the transverse left or right ("+" or "-") circular or longitudinal ("3") polarization of the phonon branches. The results are summarized in Fig. 2. There are six branches in total, namely, we have the longitudinal acoustic (LA) and longitudinal optical (LO); the transverse left-handed acoustic (TA-left) and transverse left-handed optical (TO-left); and the transverse right-handed acoustic (TA-right) and transverse right-handed optical (TO-right) modes. The associated dispersion relations take the form

$$
\left[\omega_{\alpha}^{(O / A)}\right]^{2}=\frac{1}{2 \rho j_{\alpha}}\left[b_{\alpha}+j_{\alpha} a_{\alpha} \pm \sqrt{\left(b_{\alpha}-j_{\alpha} a_{\alpha}\right)^{2}+4 j_{\alpha} \Delta_{\alpha}^{2}}\right] .
$$

The upper and lower \pm signs in the rhs of Eq. (5) correspond to the optical $(O)$ and acoustic $(A)$ branches, respectively. The $k$-dependent parameters $\left(a_{\alpha}, b_{\alpha}\right.$, and $\left.\Delta_{\alpha}\right)$ are given by $a_{ \pm}=A_{55} k^{2}, b_{ \pm}=B_{44} k^{2} \mp 2\left(C_{44}-C_{74}\right) k+$ $\left(A_{44}+A_{55}-2 A_{47}\right), \quad \Delta_{ \pm}=C_{74} k^{2} \mp\left(A_{47}-A_{55}\right) k, \quad$ and $a_{3}=A_{33} k^{2}, \quad b_{3}=B_{33} k^{2}+2\left(A_{66}-A_{69}\right), \Delta_{3}=C_{33} k^{2}$. In the long wavelength limit $k \rightarrow 0$, the frequencies of

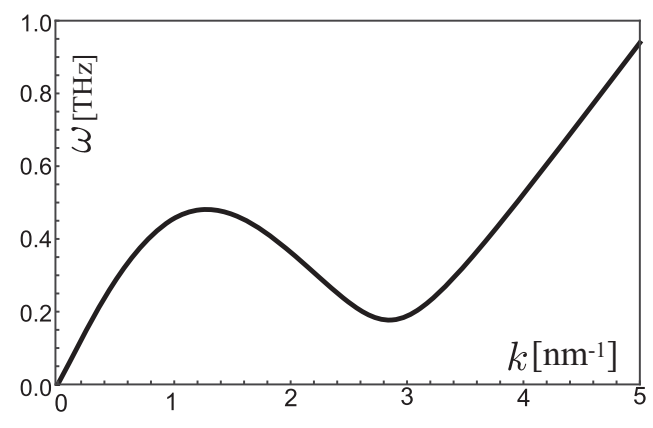

FIG. 3. The transverse right-handed acoustic (TA-right) branch that exhibits the rotonlike minimum.

the acoustic branches are proportional to the wave number, while the frequencies of the optical branches tend to finite values, $\omega_{ \pm}^{(O)}(0)=\sqrt{\left(A_{44}+A_{55}-2 A_{47}\right) / \rho j_{ \pm}}$ and $\omega_{3}^{(O)}(0)=\sqrt{\left(A_{66}-A_{69}\right) / \rho j_{3}}$.

Similarity to roton spectrum.-Figure 3 contains only the TA-right-handed phonon mode specially selected from all branches shown in Fig. 2. We observe that hybridization of the rotational and translational modes gives rise to the lowest phonon (TA-right or TA-left) branch which exhibits a roton minimum around $k_{m} \sim\left(C_{44}-C_{74}\right) / B_{44}$, reminiscent of the excitation spectrum in superfluid ${ }^{4} \mathrm{He}$ [26]. The minimum occurs around a scale inversely proportional to the unit cell length and reflects hybridization of the rotational and translational degrees of freedom of the microelement $\mathrm{CrS}_{6}$.

To elucidate reasons behind an appearance of the rotonlike minimum, we trace how translational and rotational modes contribute to the hybridized excitations. We find weights of these modes specified by the dimensionless coefficients $c_{u, \alpha}=u_{\alpha} / \sqrt{j_{\alpha}}$ and $c_{\varphi, \alpha}=\varphi_{\alpha}$, whose explicit expressions are reproduced in Supplemental Material [18]. Their $k$ dependence is illustrated by Fig. 4 for the TA-right and LA branches. It is evident that the $u_{+}-\varphi_{+}$hybridization causes resonant enhancement of the rotational degrees of freedom $\left(\varphi_{+}\right)$when approaching the roton minimum. In contrast, the ratio between the coefficients $c_{u, 3}$ and $c_{\varphi, 3}$ is reversed near the crossing of the corresponding dispersion curves, which are modified into the hybridized LA and LO phonon modes.

There is actually an interesting parallel between the TA chiral phonons and roton excitations in superfluid ${ }^{4} \mathrm{He}$. Originally, the rotons had been interpreted as a signature of some local vorticity. According to Feynman's view [26], a maximum in the static form factor $S_{q}$, which signals a short range crystalline order, gives a roton minimum in the excitation spectrum through the relation $\omega_{\boldsymbol{q}}=q^{2} / 2 m S_{\boldsymbol{q}}$. Later, Nozières [27,28] proposed an alternate scenario, where the rotons should be viewed as an incipient soft mode associated with a crystallization instability. According to this view, the Bogoliubov quasiparticles hybridize with soft density fluctuations to cause a resultant 


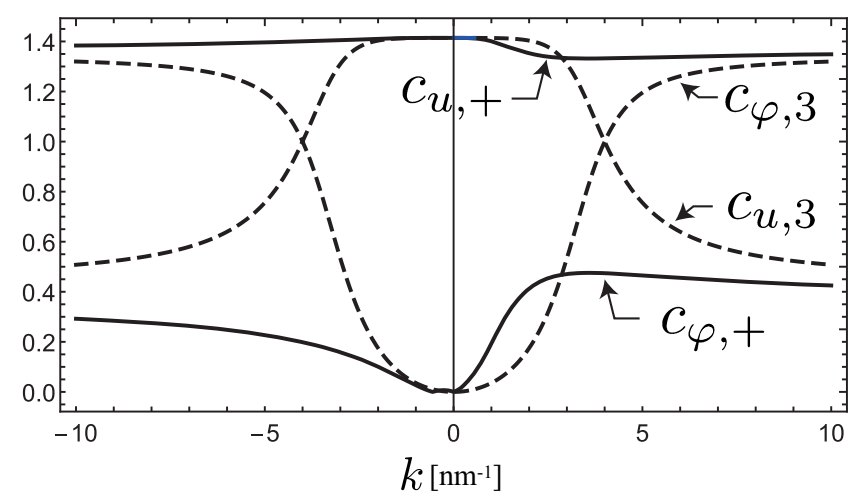

FIG. 4. The wave number dependence of the weights of translational and rotational modes in the hybridized TA-right (solid line) and LA (solid line) branches.

roton minimum. The picture can be formulated by assuming two coupled excitations where the first correspond to the Bogoliubov quasiparticles with the spectrum $\varepsilon_{q}^{B}=$ $\left[\xi_{q}^{2}+2 \xi_{q} N_{0} U\right]^{1 / 2}$ and the second due to the density fluctuation mode, which is characterized by the single mode frequency $\Omega_{q}[27,28]$. Here, $\xi_{q}$ is the boson kinetic energy, $N_{0}$ is a condensate fraction, and $U$ is a direct repulsion between bosons. The spectrum of the hybridized quasiparticles has the form similar to Eq. (5),

$$
\begin{aligned}
E_{\boldsymbol{q}}^{2}= & \frac{1}{2}\left[\Omega_{\boldsymbol{q}}^{2}+\left(\varepsilon_{\boldsymbol{q}}^{B}\right)^{2}\right] \\
& \pm \frac{1}{2}\left\{\left[\Omega_{q}^{2}-\left(\varepsilon_{\boldsymbol{q}}^{B}\right)^{2}\right]^{2}+16 \alpha_{\boldsymbol{q}}^{2} \xi_{q} \Omega_{q}\right\}^{1 / 2},
\end{aligned}
$$

where $\alpha_{q}$ is a strength of coupling between the bosons and density fluctuations. Direct comparison between Eqs. (5) and (6) eloquently illustrates the one-to-one correspondence between the translational displacement $\boldsymbol{u}(\boldsymbol{r})$ and the microrotation $\varphi(r)$ on one hand, and the Bogoliubov quasiparticles and density fluctuations on the other $\left[a_{ \pm}(k)\right.$ and $b_{ \pm}(k)$, respectively, correspond to $\varepsilon_{q}^{B}$ and $\Omega_{q}$ ].

However, the roton minimum of the superfluid helium arises in the spectrum of the longitudinal sound wave of the normal component, while in the micropolar crystal this effect is observed in propagation of the transversal elastic waves, but not for the longitudinal ones. This difference may materialize arguments propounded by Landau and Feynman that the rotons are related to local vorticity [26]. The microrotation does warrant the name roton as spinning motion of the microelement.

There is further similarity between the rotons in ${ }^{4} \mathrm{He}$ and the chiral phonons. The boson condensate lowers the roton minimum but the latter will remain finite due to the effect of condensate depletion $[27,28]$. For the chiral phonons, a finite value of the roton minimum may be inferred from the requirement that the crystal must be stable against propagating elastic waves. In Supplemental Material [18], we give proof that the stability condition implies that the roton (a)

(b)

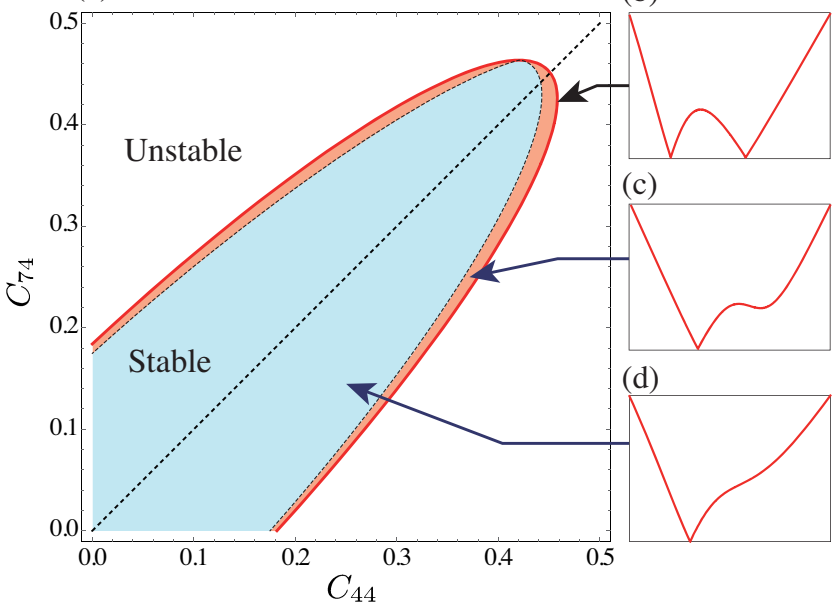

FIG. 5. (a) Phase diagram in the $C_{44}-C_{74}$ plane. The colored area corresponds to stability of the crystal structure. The roton minimum appears in the vicinity of the soft mode instability (red). The concomitant profiles of the spectrum are shown on the right (b)-(d). Numerical parameters are the same as in Fig. 2.

minimum will remain finite (never touch zero). An area of stability within the $C_{44}-C_{74}$ plane (these constants being responsible for parity breaking) is plotted in Fig. 5, together with a region where the roton minimum emerges.

The roton minimum occurs around $k \sim 3 \mathrm{~nm}^{-1}$ and roughly corresponds to $20 \mathrm{~nm}$ in real space, so the continuum theory developed here may be still be valid. The inelastic neutron or Brillouin light scattering measurements may potentially probe this finite $k$ excitation. More quantitative arguments may be required to build a bridge between the present continuum theory and atomic lattice model, which is left for future studies.

Phonon angular momentum.-Once the spectrum of the micropolar waves is known, one is capable to determine naturally orbital and spin parts of the angular momentum associated with elastic deformations. By specifying the basis of the left-handed $(L)$ and right-handed $(R)$ circularly polarized transverse modes as $\left|R_{u}\right\rangle=\left|L_{u}\right\rangle^{*}=$ $(1 / \sqrt{2})(1, i, 0,0,0,0)^{T}$, and $\left|R_{\varphi}\right\rangle=\left|L_{\varphi}\right\rangle^{*}=(1 / \sqrt{2})(0,0$, $0,1, i, 0)^{T}$ the solution $|\boldsymbol{v}\rangle^{T}=\left(c_{u, 1}, c_{u, 2}, c_{u, 3}, c_{\varphi, 1}, c_{\varphi, 2}, c_{\varphi, 3}\right)$ of Eqs. (1)-(4) may be decomposed as $|\boldsymbol{v}\rangle=$ $\sum_{\alpha=u, \varphi}\left\langle R_{\alpha} \mid v\right\rangle\left|R_{\alpha}\right\rangle+\left\langle L_{\alpha} \mid v\right\rangle\left|L_{\alpha}\right\rangle$. Then, the generator of rotations of the phonon polarization plane around the $z$ axis, or phonon spin [16], is $\hat{S}^{z}=\hbar \sum_{\alpha=u, \varphi}\left(\left|R_{\alpha}\right\rangle\right.$ $\left.\left\langle R_{\alpha}|-| L_{\alpha}\right\rangle\left\langle L_{\alpha}\right|\right)$, so that

$$
\left\langle\boldsymbol{v}\left|\hat{S}^{z}\right| \boldsymbol{v}\right\rangle=\frac{\hbar}{2} \sum_{f=u, \varphi}\left(\left|c_{f,-}\right|^{2}-\left|c_{f,+}\right|^{2}\right)
$$

is fulfilled. Obviously, the phonon circular polarization is quantized, i.e., it can take only the $\pm \hbar$ values for the “干” branches, respectively. Unlike chiral phonons in 
the theories [16,17], the phonon spin is introduced for any $k$ point of the Brillouin zone. Neglecting the microrotations $\boldsymbol{\varphi}$, the definition coincides with the spin part of the phonon angular momentum given in Ref. [29], $\boldsymbol{L}_{s}=\hbar \sum_{k} \boldsymbol{e}_{\boldsymbol{k}}\left(a_{\boldsymbol{k}-}^{\dagger} a_{k-}-a_{\boldsymbol{k}+}^{\dagger} a_{k+}\right)$, where the angular momenta $\hbar$ of the individual $(\boldsymbol{k} \pm)$ phonons add up either parallel or antiparallel to their wave vectors, $\boldsymbol{e}_{\boldsymbol{k}}=\boldsymbol{k} /|\boldsymbol{k}|$. The classical interpretation of the resultant $\boldsymbol{L}_{s}$ corresponds to small-radius circular shear displacements of points around their equilibrium positions [Fig. 1(b)].

With regard to the orbital part of the phonon angular momentum, its definition, $L_{ \pm}=\rho j_{ \pm} \dot{\varphi}_{ \pm}$, follows directly from the appropriate conservation law of the micropolar theory [10]. This implies propagation of plane waves of the angular momentum density.

Roton and acoustic activity.-Nonreciprocity of the chiral phonons results in consequential acoustic activity, most notably in the vicinity of the roton minimum. By definition, the effect occurs when incident transverse acoustic waves, which are linearly polarized, propagate in the crystal along the $z$ axis. It is characterized by the rotation angle, $\phi=\frac{1}{2} \omega l\left(1 / v_{-}-1 / v_{+}\right)$, at a distance $l$ from the incident surface. The phase velocities $v_{ \pm}$of the circularly polarized transverse modes may be calculated from the dispersion relations (5) to give

$$
\begin{aligned}
\frac{\phi}{l}= & \rho \omega^{2} \frac{\left(A_{47}-A_{55}\right)}{\left(A_{44} A_{55}-A_{47}^{2}\right)^{2}} \\
& \times\left[A_{55} C_{44}+A_{44} C_{74}-A_{47}\left(C_{44}+C_{74}\right)\right] .
\end{aligned}
$$

Comparison with EOMs, Eqs. (1) and (2), shows that apart from the intercross coupling $A_{55}-A_{47}$ between the microtranslations and microrotations, the inherent gyrotropy $C_{44}-C_{74}$ of the microrotations is an essential element of acoustic activity. Unlike the micropolar theory, the conventional approach [5,6] relates the latter to nonlocal interaction between stress and strain; this is reflected in the first-order dispersion in expansion of the elastic coefficients $c_{i j}(\boldsymbol{k}, \omega)$. As a consequence, a difference in phase velocities of circularly polarized waves appears at any $\boldsymbol{k}$ vector.

Concluding remarks.-We demonstrate polarizationdependent splitting of phonon bands in a chiral crystal, using the micropolar elasticity theory for $\mathrm{CrNb}_{3} \mathrm{~S}_{6}$. Our main results may be summarized as follows. (I) The splitting is reached solely within the phonon sector of elementary excitations, and it is maintained by coupling between the transverse translational and rotational modes of the micropolar medium. (II) Transverse acoustic branches of the hybridized phonon spectrum exhibit a roton minimum reminiscent of elementary excitations in the superfluid helium- 4 . We argue that the translational and rotational degrees of freedom of the chiral phonon system correspond to the Bogoliubov quasiparticles and massive density fluctuations, respectively, in the superfluid helium4. In addition, we discuss acoustic activity originating from nonreciprocity of the phonon spectrum. It is expected that the polarization-dependent phonon dispersions demonstrated may lead to chirality-induced cross correlations among lattice, electronic, and magnetic degrees of freedom.

The authors express special thanks to Yusuke Kato for directing our attention to Refs. [27,28]. We thank Laurence Barron for continuous encouragement. We also thank Nikolay Baranov, Yoshihiko Togawa, and Hiroshi Yamamoto for stimulating discussions concerning experimental insights. The authors acknowledge JSPS Bilateral Joint Research Projects (JSPS-RFBR), the Russian Foundation for Basic Research (RFBR), Grant No. 2052-50005. This work was supported by JSPS KAKENHI Grant No. 17H02923. A. S. O. acknowledges funding by Act 211 Government of the Russian Federation, Contract No. 02.A03.21.0006, and the Ministry of Education and Science of Russia, Project No. FEUZ-2020-0054. A. A. T. acknowledges the financial support of Competitiveness Enhancement Program CEP 3.1.1.1-20.

[1] A. A. Bukharaev, A. K. Zvezdin, A. P. Pyatakov, and Y. K. Fetisov, Phys. Usp. 61, 1175 (2018).

[2] L. D. Barron, Chirality 24, 879 (2012).

[3] L. D. Barron, Molecular Light Scattering and Optical Activity, 2nd ed. (Cambridge University Press, Cambridge, England, 2004).

[4] T. Frenzel, J. Köfler, E. Jung, M. Kadic, and M. Wegener, Nat. Commun. 10, 3384 (2019); I. Fernandez-Corbaton, C. Rockstuhl, P. Ziemke, P. Gumbsch, A. Albiez, R. Schwaiger, T. Frenzel, M. Kadic, and M. Wegener, Adv. Mater. 31, 1807742 (2019).

[5] D. L. Portigal and E. Burstein, Phys. Rev. 170, 673 (1968).

[6] A. S. Pine, J. Acoust. Soc. Am. 49, 1026 (1971).

[7] J. Kishine and A. S. Ovchinnikov, Solid State Phys. 66, 1 (2015).

[8] G. W. Paterson, A. A. Tereshchenko, S. Nakayama, Y. Kousaka, J. Kishine, S. McVitie, A. S. Ovchinnikov, I. Proskurin, and Y. Togawa, Phys. Rev. B 101, 184424 (2020).

[9] L.D. Landau and E. M. Lifshitz, Theory of Elasticity (Butterworth-Heinemann, Oxford, 1986).

[10] A. C. Eringen, Microcontinuum Field Theories: I. Foundations and Solids (Springer-Verlag, New York, 1999).

[11] W. Nowacki, Theory of Asymmetric Elasticity (Pergamon Press, Oxford, 1986).

[12] J. Pouget, A. Aşkar, and G. A. Maugin, Phys. Rev. B 33, 6304 (1986); 33, 6320 (1986).

[13] E. I. Rashba and V. I. Sheka, Fiz. Tverd. Tela 1, 162 (1959) (in Russian); G. Bihlmayer, O. Rader, and R. Winkler, New J. Phys. 17, 050202 (2015).

[14] A. A. Tereshchenko, A. S. Ovchinnikov, I. Proskurin, E. V. Sinitsyn, and J. I. Kishine, Phys. Rev. B 97, 184303 (2018).

[15] T. Nomura, X.-X. Zhang, S. Zherlitsyn, J. Wosnitza, Y. Tokura, N. Nagaosa, and S. Seki, Phys. Rev. Lett. 122, 145901 (2019). 
[16] L. Zhang and Q. Niu, Phys. Rev. Lett. 115, 115502 (2015); H. Zhu, J. Yi, M.-Y. Li, J. Xiao, L. Zhang, C.-W. Yang, R. A. Kaindl, L.-J. Li, Y. Wang, and X. Zhang, Science 359, 579 (2018).

[17] H. Chen, W. Wu, S. A. Yang, X. Li, and L. Zhang, Phys. Rev. B 100, 094303 (2019).

[18] See Supplemental Material at http://link.aps.org/ supplemental/10.1103/PhysRevLett.125.245302 for technical details related to the micropolar elasticity theory, which includes Refs. [19,20].

[19] H. A. Jahn, Acta Crystallogr. 2, 30 (1949).

[20] Y. I. Sirotin and M. P. Shaskolskaya, Fundamentals of Crystal Physics (Mir, Moscow, 1982).

[21] L. M. Volkova and D. V. Marinin, J. Appl. Phys. 116, 133901 (2014).
[22] P. R. Sarode, Phys. Status Solidi A 98, 391 (1986).

[23] N. J. Ghimire, M. A. McGuire, D. S. Parker, B. Sipos, S. Tang, J.-Q.Yan, B. C. Sales, and D. Mandrus, Phys. Rev. B 87, 104403 (2013).

[24] The order of the microinertia tensor is ensued from the definition $j_{k l}=\left\langle\xi_{k} \xi_{l}\right\rangle$, where $\xi$ is the microdeformation vector (i.e., see Ref. [10]).

[25] R. Aoki, Y. Kousaka, and Y. Togawa, Phys. Rev. Lett. 122, 057206 (2019).

[26] L. Landau, Phys. Rev. 60, 356 (1941); R. P. Feynman, Rev. Mod. Phys. 29, 205 (1957).

[27] P. Nozières, J. Low Temp. Phys. 137, 45 (2004).

[28] P. Nozières, J. Low Temp. Phys. 142, 91 (2006).

[29] D. A. Garanin and E. M. Chudnovsky, Phys. Rev. B 92, 024421 (2015). 